Check for updates

Cite this: RSC Adv., 2018, 8, 23742

Received 1st June 2018

Accepted 24th June 2018

DOI: $10.1039 / \mathrm{c} 8 \mathrm{ra04690e}$

rsc.li/rsc-advances

\section{Photoinduced viscosity control of lecithin-based reverse wormlike micellar systems using azobenzene derivatives $\uparrow$}

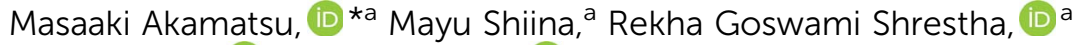 \\ Kenichi Sakai, (iD ab Masahiko Abe (iD b and Hideki Sakai*ab
}

\begin{abstract}
This report describes the controlled viscosity changes of photoresponsive reverse wormlike micellar systems formed by soybean lecithin (SoyPC), D-ribose, and azobenzene derivatives in decane. UV light irradiation produces a significant ( 150 -fold) decrease in solution viscosity by triggering a structural transformation of the wormlike micelles. Subsequent visible light irradiation leads to recovery of the initial micellar structure and elevated solution viscoelasticity. This dramatic, reversible variation in solution viscosity by light irradiation can be applied to cosmetics, personal care products, and device components.
\end{abstract}

\section{Introduction}

Wormlike micelles are polymer-like molecular assemblies characterized by an elevated solution viscoelasticity due to micellar entanglement in an aqueous medium. ${ }^{1}$ The characteristic structure of wormlike micelles forms when the critical packing parameter (CPP), which is defined as the ratio of the volume of the hydrophobic group (v) to the product of the length $(l)$ of this group and the cross-sectional area $(a)$ of the hydrophilic group $(\mathrm{CPP}=v / a l)$, is approximately $1 / 2$. Appropriate packing of surfactants leads to the formation of wormlike micelles. Aqueous wormlike micelle solutions have applications in cosmetics, personal care products, and paints, because of their solubilization behaviour and tunable viscoelasticity. On the other hand, reverse wormlike micelles, which are formed in organic solvents, extend these applications to conditions that are incompatible with water (e.g., extreme temperatures and pressures) or can regulate the drying speed of materials. Most reverse wormlike micelles are formed by mixtures of biocompatible lecithin and polar additives, such as water, bile salts, and glycerols. ${ }^{2}$ The additives reduce electrostatic repulsions between the lecithin head groups and induce formation of the wormlike micelles.

Controlled formation of micelles by external stimuli, such as light or magnetic field irradiation, a redox reaction, or a temperature or $\mathrm{pH}$ change, enables the modulation of solution properties. ${ }^{3}$ Light is a promising stimulus, because it is clean and easily applied with high spatial resolution. Photoresponsive micelles

${ }^{a}$ Department of Pure and Applied Chemistry, Faculty of Science and Technology, Tokyo University of Science, 2641 Yamazaki, Noda, Chiba 278-8510, Japan

${ }^{b}$ Research Institute for Science and Technology, Tokyo University of Science, 2641 Yamazaki, Noda, Chiba 278-8510, Japan

$\dagger$ Electronic supplementary information (ESI) available. See DOI: 10.1039/c8ra04690e have applications in the controlled release of perfumes and drugs. For example, Eastoe and Abbott demonstrated that vesicles formed by bolaform or gemini surfactants containing a photoresponsive azobenzene or a stilbene unit transformed into micelles upon UV irradiation., ${ }^{4,5}$ We have reported the photochemical control of micellar structure and release of an oily substance upon changes in solubilization amounts in a system based on a cationic azobenzene-type surfactant..$^{6-9}$

We also have demonstrated photoinduced viscosity changes of an wormlike micellar solution in an aqueous medium with a photoresponsive surfactant ${ }^{10,11}$ or an additive. ${ }^{12}$ In related studies, Zakin, Raghavan and co-workers demonstrated a highly efficient regulation of heat transfer properties of aqueous solutions of photoresponsive wormlike micelles. ${ }^{13,14}$ This discovery opens the possible application of wormlike micelles as device components. If photoirradiation can control the formation of reverse wormlike micelles in non-aqueous media, the approach can be applied in organic media and thus be used to control heat-transfer capacities at extreme temperatures and pressures and the drying speed of paints and related materials. Development of photoresponsive reverse wormlike micelles is required to achieve this goal. The photoswitchable reverse wormlike micelles have been developed with mixtures of biocompatible lecithin and photoresponsive additives ${ }^{15-17}$ or synthetic surfactants. ${ }^{18}$ For instance, Raghavan et al. accomplished reversible viscosity changes of an organic solvent containing a reverse wormlike micellar system of spiropyran/soybean lecithin (SoyPC)/sodium deoxycholate/cyclohexane. ${ }^{15}$ Later on, our groups demonstrated larger viscosity changes in oil using biologically-originated cinnamic acid derivatives as photoswitchable additives, ${ }^{\mathbf{1 6 , 1 7}}$. However, this photoinduced viscosity change is unidirectional due to irreversible photoisomerization of the cinnamic acid derivatives.

We demonstrate herein dramatic and reversible variations in solution viscosity of photoresponsive reverse wormlike micelles 
comprising a biocompatible surfactant (SoyPC) and a simple azobenzene derivative. To achieve this goal, we chose the reverse wormlike formed by D-ribose/SoyPC/decane reported by Hashizaki et al. ${ }^{19}$ Addition of D-ribose to a decane solution of SoyPC forms spherical or ellipsoidal reverse micelles, which then grow into reverse wormlikes. D-ribose molecules contain hydroxyl groups that hydrogen bond to the phosphate groups of SoyPC, which reduces the curvature of the micelles and promotes the structural transformation. To achieve reversible photoswitching of the micellar structure, azobenzene derivatives that isomerize between trans and cis forms upon ultraviolet (UV) and visible light irradiation (Scheme 1a) were added to the solution of the lecithin-based reverse wormlike micelles. The effects of photoisomerization and polar substituents on azobenzene derivatives on viscosity changes were examined systematically. The dramatic reversible viscosity changes using SoyPC and simple azobenzene derivatives in a non-aqueous medium promises broad application.

\section{Experimental}

\subsection{General procedures}

Solvents and reagents were purchased from Tokyo Chemical Industry Co., Ltd. (Tokyo, Japan) or Wako Pure Chemical Co. (Osaka, Japan) and were used without further purification. 4Butoxyaniline was purchased from Wako Pure Chemical Co. (Osaka, Japan). L- $\alpha$-phosphatidylcholine (SoyPC, 95\%) was purchased from Avanti Polar Lipids, Inc. (Alabama, US). This product has saturated/unsaturated hydrocarbon chains of several lengths. The chemical structure shown in Scheme 1 is the major component of SoyPC (95\%). D-ribose was purchased from Wako Pure Chemical Co. (Osaka, Japan). Azobenzene derivatives (4-7) were purchased from Tokyo Chemical Industry Co., Ltd. (Tokyo, Japan). All reaction mixtures and fractions eluted by column chromatography were monitored using thin layer chromatography (TLC) plates (Merck, Kieselgel 60 F254).

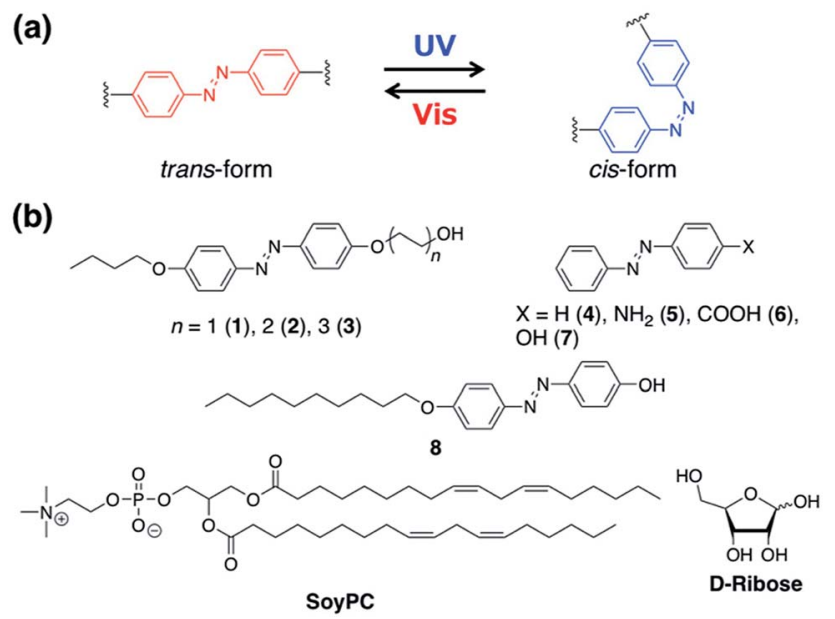

Scheme 1 (a) Structural changes of the azobenzene moiety induced by UV and visible light irradiations. (b) Molecular structures of synthesized azobenzene derivatives $(1-3,8)$, azobenzene with and without polar substituents (4-7), SoyPC, and D-ribose.
The TLC plates were examined under UV light at 254 and $365 \mathrm{~nm}$. Flash column chromatography over silica gel (Wakosil C-200, 64-210 $\mu \mathrm{m}$ ) was used for all separations.

\subsection{Measurements}

${ }^{1} \mathrm{H}$ - and ${ }^{13} \mathrm{C}-\mathrm{NMR}$ spectra were measured at $298 \mathrm{~K}$ in $\mathrm{CDCl}_{3}$ solutions using a JEOL model JNM-AL300 (300 MHz) spectrometer with $\mathrm{Si}\left(\mathrm{CH}_{3}\right)_{4}$ as internal standard. Chemical shifts $(\delta)$ and coupling constants $(J)$ are given in parts per million (ppm) and Hertz $(\mathrm{Hz})$, respectively. ESI-MS spectra were obtained with a JASCO model JMS-T100CS spectrometer. UV/vis absorption spectra were measured using an Agilent 222 UV/vis spectrophotometer with a quartz cuvette $(1.0 \mathrm{~cm}$ path length). Static and dynamic rheological measurements were carried out with a TA Instruments AR-G2 unit using cone-plate geometries $(40 \mathrm{~mm}$ diameter with a $2^{\circ}$ cone angle). Frequency sweep measurements were performed in the linear viscoelastic regime of the samples, as previously determined by dynamic strain sweep measurements. Infrared absorption spectra (at $1 \mathrm{~cm}^{-1}$ resolution) were recorded with a JASCO model FT/IR-6100 spectrometer. Measurements were performed by the liquid membrane method using $\mathrm{NaCl}$ plates. Optical microscopic images were obtained with an IMT-2 microscope (Olympus Optical Co., Ltd.). Polarized optical micrographs were observed through crossed polarizers. The resulting images were transferred to a computer equipped with a Moticam 2000 digital camera fitted on the eyepiece.

\subsection{Preparation of reverse wormlike micelle solutions}

SoyPC (105 $\mathrm{mM})$ and D-ribose $(55 \mathrm{mM})$ were dissolved in methanol. The solvent was removed under reduced pressure, and the residue was kept in a vacuum desiccator for 3 days to dry the mixture completely. Decane was added to the resulting mixture, which was stirred for 1 day to obtain a homogenous reverse wormlike micelle solution. Azobenzene derivatives (1-7) were added to the micellar solution, which was heated at $60{ }^{\circ} \mathrm{C}$ to achieve homogeneity and then stirred by a vortex mixer for $1 \mathrm{~min}(2500 \mathrm{rpm})$. The micelle solutions containing azobenzene derivatives were maintained at room temperature for at least 1 day to reach equilibrium.

\subsection{UV and visible light irradiation}

UV and visible light irradiations were conducted using a $200 \mathrm{~W}$ Hg-Xe lamp (Supercur UVF-203S, San-Ei Electronic). Irradiation wavelength ranges (UV: 260-390 nm; visible: $>420 \mathrm{~nm}$ ) were obtained using U-340 and L-42 colour filters supplied by the Hoya Candeo Optronics Corp. for UV and visible light, respectively. Each solution was irradiated at $100 \mathrm{~mW} \mathrm{~cm}^{-2}$ in a $1.0 \mathrm{~cm}$ pathlength quartz cuvette until photostationary states were observed in the UV/vis absorption spectrum.

\subsection{Synthesis}

Syntheses of azobenzene derivatives (1-3) were carried out with 4-((4-butoxyphenyl)diazenyl)phenol as starting material, which was prepared according to the literature. ${ }^{20}$ Derivative 8 was synthesized by a published procedure. ${ }^{21}$ 
2-(4-((4-Butoxyphenyl)diazenyl)phenoxy)ethan-1-ol (1). $\mathrm{K}_{2} \mathrm{CO}_{3}$ $(1.58 \mathrm{~g}, 11.5 \mathrm{mmol})$ was added to a solution mixture of $4-((4-$ butoxyphenyl)diazenyl)phenol (1.25 g, $4.58 \mathrm{mmol}), 2$-bromoethanol (1.15 g, $9.16 \mathrm{mmol}$ ), and catalytic amounts of KI in acetone $(10 \mathrm{~mL})$. The resulting mixture was refluxed overnight. The reaction mixture was allowed to cool to room temperature, and the solid residue was filtered. The filtrate was dried over anhydrous $\mathrm{Na}_{2} \mathrm{SO}_{4}$, and the solvent was removed under reduced pressure. The crude product was recrystallized from ethyl acetate to obtain pure $1(0.128 \mathrm{~g}, 9 \%)$ as a brown powder. Synthesis of compounds 2 and 3 were carried out by same procedure. ${ }^{1} \mathrm{H}-\mathrm{NMR}$ $\left(300 \mathrm{MHz}, \mathrm{CDCl}_{3}, 25^{\circ} \mathrm{C}\right): \delta=0.99(\mathrm{t}, 3 \mathrm{H}, J=6.0 \mathrm{~Hz}), 1.45-1.58(\mathrm{~m}$, $2 \mathrm{H}), 1.76-1.85(\mathrm{~m}, 2 \mathrm{H}), 3.98-4.06(\mathrm{~m}, 4 \mathrm{H}), 4.16(\mathrm{t}, 2 \mathrm{H}, J=6.0 \mathrm{~Hz})$, 6.97-7.03 (m, 4H), 7.85-7.89 (m, 4H) ppm. ${ }^{13} \mathrm{C}-\mathrm{NMR}(75 \mathrm{MHz}$, $\left.\mathrm{CDCl}_{3}, 25{ }^{\circ} \mathrm{C}\right): \delta=14.0,19.4,31.4,61.5,68.2$, 69.6, 114.8, 114.9, $124.5,124.5,147.0,147.5,160.6,161.4$ ppm. ESI-MS: $m / z=337$ $[\mathrm{M}+\mathrm{Na}]^{+}, 651[2 \mathrm{M}+\mathrm{Na}]^{+}$.

4-(4-((4-Butoxyphenyl)diazenyl)phenoxy)butan-1-ol (2). ${ }^{1} \mathrm{H}$ NMR (300 MHz, $\left.\mathrm{CDCl}_{3}, 25{ }^{\circ} \mathrm{C}\right): \delta=0.99(\mathrm{t}, 3 \mathrm{H}, J=6.0 \mathrm{~Hz})$, 1.45-1.58 (m, 2H), 1.73-1.85 (m, 4H), 1.88-1.97 (m, 2H), 3.74$3.76(\mathrm{~s}, 2 \mathrm{H}), 4.01-4.10(\mathrm{~m}, 4 \mathrm{H}), 6.92-7.00(\mathrm{~m}, 4 \mathrm{H}), 7.84-7.88(\mathrm{~m}$, $4 \mathrm{H}) \mathrm{ppm} .{ }^{13} \mathrm{C}-\mathrm{NMR}\left(75 \mathrm{MHz}, \mathrm{CDCl}_{3}, 25{ }^{\circ} \mathrm{C}\right): \delta=14.0,19.4,25.9$, 29.5, 31.4, 62.6, 68.2, 114.8, 114.8, 124.4, 147.0, 147.2, 161.0, 161.4 ppm. ESI-MS: $m / z=365[\mathrm{M}+\mathrm{Na}]^{+}, 707[2 \mathrm{M}+\mathrm{Na}]^{+}$.

6-(4-((4-Butoxyphenyl)diazenyl)phenoxy)hexan-1-ol (3). ${ }^{1} \mathrm{H}$ NMR $\left(300 \mathrm{MHz}, \mathrm{CDCl}_{3}, 25{ }^{\circ} \mathrm{C}\right): \delta=0.99(\mathrm{t}, 3 \mathrm{H}, J=6.0 \mathrm{~Hz}), 1.38$ $1.55(\mathrm{~m}, 6 \mathrm{H}), 1.56-1.66(\mathrm{~m}, 2 \mathrm{H}), 1.75-1.87(\mathrm{~m}, 4 \mathrm{H}), 3.66(\mathrm{t}, 2 \mathrm{H}, J=$ $6.0 \mathrm{~Hz}), 4.00-4.05(\mathrm{~m}, 4 \mathrm{H}), 6.96-7.00(\mathrm{~m}, 4 \mathrm{H}), 7.84-7.88(\mathrm{~m}$, $4 \mathrm{H})$ ppm. ${ }^{13} \mathrm{C}-\mathrm{NMR}\left(75 \mathrm{MHz}, \mathrm{CDCl}_{3}, 25{ }^{\circ} \mathrm{C}\right): \delta=14.0,19.4,25.7$, 26.0, 29.3, 31.4, 32.8, 63.0, 68.1, 68.3, 114.8, 124.4, 147.0, 147.1, 161.2, 161.3 ppm. ESI-MS: $m / z=393[\mathrm{M}+\mathrm{Na}]^{+}, 763[2 \mathrm{M}+\mathrm{Na}]^{+}$.

\section{Results and discussion}

\subsection{Effect of the alkyl chain length of azobenzene} derivatives on photoresponsive solution viscosity

Azobenzene derivatives bearing a polar hydroxylalkyl with different chain lengths and a butoxy group were synthesized (13). Alkyl chains were introduced to the azobenzene core to enhance the affinity for lipids. A mixture of SoyPC (105 mM) and
D-ribose $(55 \mathrm{mM})$ in decane forms reverse wormlike micelles. ${ }^{19}$ The zero-shear viscosity, $\eta_{0}$, of the solution was $200 \mathrm{~Pa}$ s. Azobenzene derivatives (1-3) at a fixed concentration of $15 \mathrm{mM}$ were added to the micelle solution. The viscosity decreased slightly in comparison with the original solution following addition of derivatives 2 and 3 (Fig. 1a). On the other hand, introduction of $\mathbf{1}$ bearing a shorter alkyl group showed a significant decrease in $\eta_{0}$, which indicates a transition from reverse wormlike micelles to shorter ones or rodlike micelles.

To confirm the photoreactivity of the micellar solutions, UV light was irradiated to the mixtures to induce the trans-to-cis photoisomerization of 1-3. After UV irradiation, the zero-shear viscosity of all samples decreased. The solution of 2 gave the greatest decrease in viscosity from 101 to $0.7 \mathrm{~Pa} \mathrm{~s}$ (approximately a 150-fold change). Steady-state rheology measurements on the solution of 2 showed a Newtonian region at low shear rates, where viscosity is constant and independent of the applied perturbation, and a non-Newtonian region at higher shear rates, where viscosity is dependent on the applied shear (Fig. 1b). This so-called shear-thinning behaviour is typical of wormlike micellar aggregates. After UV irradiation, a Newtonian response was observed over the entire frequency range. Subsequent visible light irradiation restored the original viscosity and shear-thinning, which means that visible light induces reformation of the reverse wormlike micelles by means of the cis-totrans photoisomerization. These results demonstrate that a $2 / \mathrm{D}^{-}$ ribose/SoyPC/decane solution of reverse wormlike micelles undergoes significant and reversible viscosity changes upon UV and subsequent visible light irradiation.

Dynamic rheology experiments were performed before and after irradiation to characterize the structure of the reverse wormlike micelles. Fig. 1c shows dynamic rheological responses in elastic modulus $\left(G^{\prime}\right)$ and viscous modulus $\left(G^{\prime \prime}\right)$ for the micellar solution of 2 as a function of oscillation frequency $(\omega)$. The data before irradiation show a typical viscoelastic response, which is viscous-dominant $\left(G^{\prime \prime}>G^{\prime}\right)$ at low frequencies and elastic-dominant $\left(G^{\prime}>G^{\prime \prime}\right.$, plateau in $\left.G^{\prime}\right)$ at high frequencies. The intersection of $G^{\prime}$ and $G^{\prime \prime}$ defines the relaxation time, $\tau_{\mathrm{R}}$, of $1.39 \mathrm{~s}$. The constant value of $G^{\prime}$ at high frequencies (87.9 $\mathrm{Pa} \mathrm{s}$ in Fig. 1c) is called the plateau modulus, $G_{0}$, which
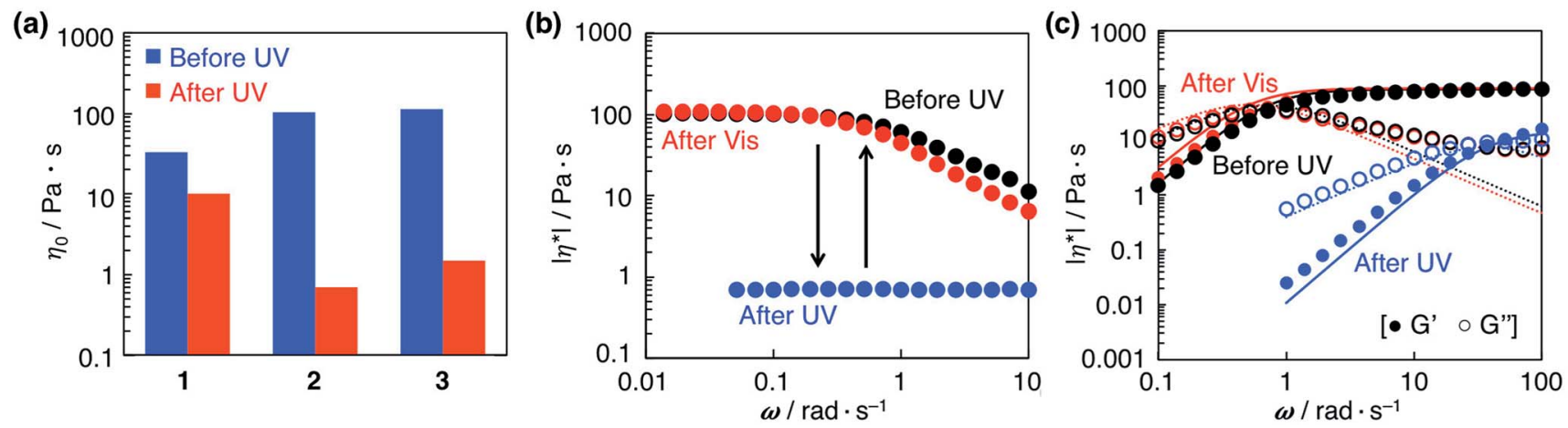

Fig. 1 Zero-shear viscosity, $\eta_{0}$, of 15 mM 1-3/55 mM D-ribose/105 mM SoyPC/decane mixtures before and after UV irradiation (a). Steady-state rheology of a 2/D-ribose/SoyPC/decane mixture before (black) and after UV (blue) and subsequent visible light irradiation (red) (b). Variations in elastic modulus, $G^{\prime}$ (filled circle), and viscous modulus, $G^{\prime \prime}$ (open circle), as a function of oscillation frequency, $\omega$, of a 2/D-ribose/SoyPC/decane mixture before (black) and after UV (blue) and subsequent visible light irradiation (red) (c). 
reflects the extent of entanglement of wormlike micelles. The experimental data fit with the Maxwell model, ${ }^{22}$ which confirms the existence of entangled wormlike micelles. However, deviation of experimental plots of $G^{\prime \prime}$ from the theoretical curves is observed at high frequencies. This behaviour can be explained by the presence of faster Rouse modes in addition to the slower reptational relaxations of the Maxwell model. ${ }^{20} \mathrm{UV}$ irradiation shifts the intersection of $G^{\prime}$ and $G^{\prime \prime}$ to higher frequencies and produces a shorter relaxation time $\left(\tau_{\mathrm{R}}=0.268 \mathrm{~s}\right)$, which indicates that relaxation against shear stress occurs within shorter time scales after UV irradiation. Moreover, the smaller value of $G_{0}(15.9 \mathrm{~Pa}$ s) corresponds to a decrease in entanglement of the reverse wormlike micelles. Subsequent irradiation with visible light leads to recovery of the initial $G^{\prime}$ and $G^{\prime \prime}$ curves. These results confirm that photoirradiation produces a reversible morphological transformation of the reverse wormlike micelles.

\subsection{Reversibility of changes in solution viscosity with photoirradiation}

The continuous viscosity measurements illustrated in Fig. 2a demonstrate that the dramatic variations in $\eta_{0}$ upon UV and subsequent visible light irradiation are reversible. Photoisomerization of azobenzene derivatives was monitored by UV/ vis spectroscopy. As shown in Fig. $2 \mathrm{~b}$ and $\mathrm{S} 1, \dagger$ the $350 \mathrm{~nm}$ absorption maximum of trans-azobenzene decreases, and a broad band at $430 \mathrm{~nm}$ originating from cis-azobenzene appears upon UV irradiation. Deconvolution of the spectrum shows that approximately $45 \%$ of the trans form isomerizes to the cis form upon UV irradiation. Subsequent visible light irradiation results in recovery of the peak at $350 \mathrm{~nm}$ and returns all but $9 \%$ of the total azobenzene to the original trans configuration. These results demonstrate that the trans-cis photoisomerization of azobenzene is consistent with the reversible changes in solution viscosity.
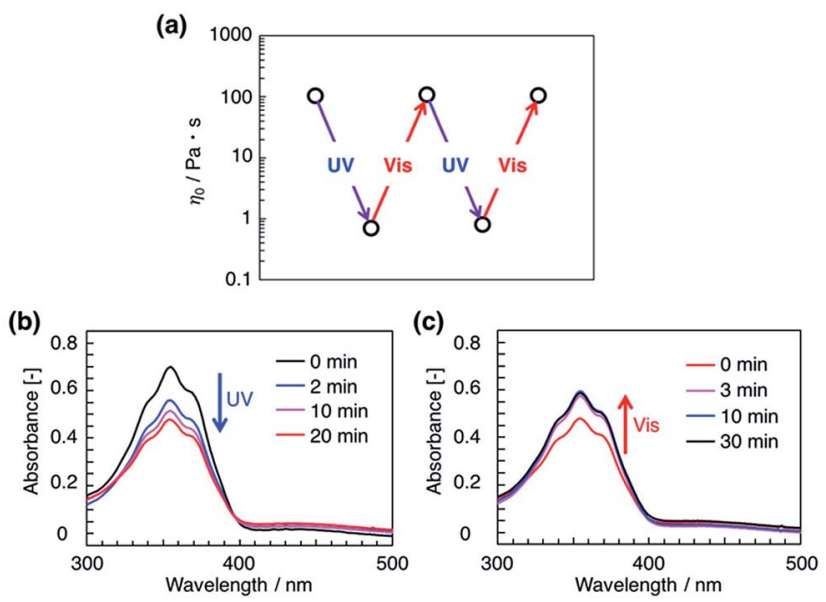

Fig. 2 Repeatability of zero-shear viscosity changes of a $15 \mathrm{mM} \mathrm{2/}$ $55 \mathrm{mM}$ D-ribose/105 mM SoyPC/decane mixture upon UV and subsequent visible light irradiation (a). UV/vis absorption spectra of solutions after (b) UV and (c) subsequent visible light irradiation (the 2/ D-ribose/SoyPC/decane mixtures were diluted 400-fold with decane before the measurements).

\subsection{Effect of substituents on azobenzene derivatives to the photorheological responses}

Viscosity data for reverse wormlike micelle solutions with a series of azobenzene derivatives (4-8) are shown in Fig. 3.

Addition of non- or primary amine-substituted azobenzene (4 or 5) to a D-ribose/SoyPC/decane mixture produces viscosity values similar to that of 2 . UV irradiation of these samples induces a decrease in solution viscosity, but with differences less than that of 2. Addition of azobenzene analogues bearing a carboxylic or a hydroxyl group (6 or 7) without light irradiation leads to a large decrease in $\eta_{0}$, which suggests that interaction of strongly polar substituents with the headgroups of SoyPC disrupts formation of reverse wormlike micelles. Infrared absorption spectra of decane solutions containing 6 and 7 show downshifts of a band originating from the stretching vibration of the $\mathrm{P}=\mathrm{O}$ group in SoyPC (Fig. S2 $\dagger$ ), which suggests that the azobenzene derivatives bearing a carboxylic or a hydroxyl group strongly penetrate into the micelles and changes the micellar structures.

Addition of an azobenzene derivative bearing both a hydroxyl group and a long alkyl group (8) gives a smaller decrease in solution viscosity than 7 , which indicates that the enhanced hydrophobicity of $\mathbf{8}$ weakens crucial effect of the phenolic group on formation of the reverse wormlike micelles. UV irradiation of the sample causes an approximately 8-fold decrease in $\eta_{0}$. Taking into account the polarity and the position of the substituents on azobenzene core, the weakly polar alcoholic group and the less bulky alkyl chain next to the alcoholic group on 2 maintains formation of reverse wormlike micelles after the addition of azobenzene derivative, compared with the phenolic group on 8.

These results indicate that an azobenzene derivative with appropriate hydrophobicity is required to maintain viscosity of the original D-ribose/SoyPC/decane mixture and produce a large change of solution viscosity upon UV irradiation. The behaviours of $\mathbf{1}$ and $\mathbf{3}$ with shorter or longer alkyl chain support this interpretation. It is also found that hydrophobicity of the azobenzene analogs governs its solubilization position in the micellar solutions and impacts of light irradiation on structure of the micelles.

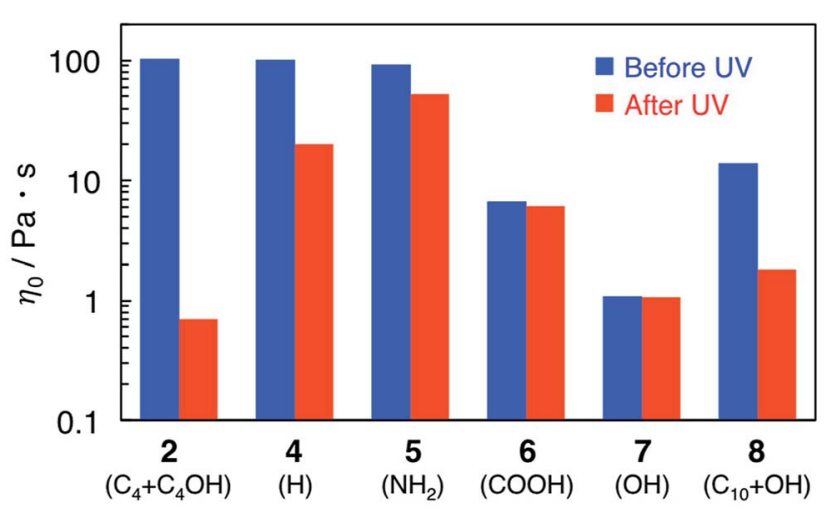

Fig. 3 Zero-shear viscosity, $\eta_{0}$, for $15 \mathrm{mM}$ azobenzene derivatives (2, 4-8)/55 mM D-ribose/105 mM SoyPC/decane mixtures before and after UV irradiation. 
(a)

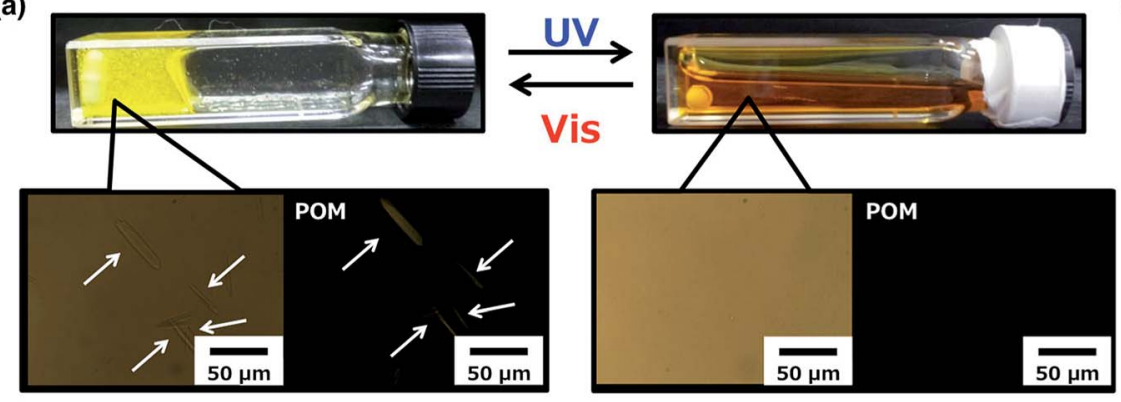

(b)

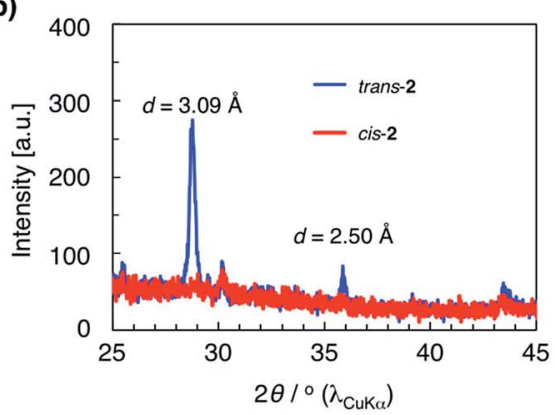

Fig. 4 Photographs, optical micrographs, and polarized optical micrographs (POM) of a $15 \mathrm{mM}$ 2/D-ribose/SoyPC/decane mixture before and after UV light irradiation (a). Powder X-ray diffraction patterns of trans- and cis-2 (b).

\subsection{Impact of isomerization of azobenzene derivatives on reverse wormlike micellar structure}

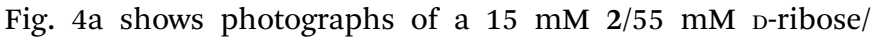
$105 \mathrm{mM}$ SoyPC/decane mixture. Before UV irradiation, the solution does not flow when the sample cell is tilted, because entangled wormlike micelles are present. Exposure to UV light significantly decreases the solution viscosity causing the sample to become liquid-like. As shown on the left of Fig. 4, solid particles are observed in solution before irradiation. The particles are needle-like and show birefringence by polarized optical microscopy (Fig. 4a), which suggests that the trans form of $\mathbf{2}$ has poor solubility and a part of them exists as a crystalline solid in decane. The poor solubility of trans-2 in decane is also observed in Fig. $\mathrm{S} 3 \uparrow$ (approximately $70 \mu \mathrm{M}$ ). These particles disappear from the micrograph after UV irradiation, indicating that the cis form of 2 is soluble in decane (Fig. 4a, right). An Xray diffraction profile of a solid sample of the trans form of 2 contains peaks, from which the calculated $d$-spacing corresponds to the characteristic length of $\pi-\pi$ stacking $^{23,24}$ (Fig. $4 \mathrm{~b}$ ). These peaks disappear after UV irradiation, indicating that the cis form of 2 lacks these intermolecular interactions and exhibits good solubility in the reverse wormlike micellar solution.

Based on these results, we propose that the changes in solution viscosity upon photoirradiation occur by the following mechanism (Fig. 5). When the azobenzene derivative 2 is added into a D-ribose/SoyPC/decane mixture, formation of the reverse
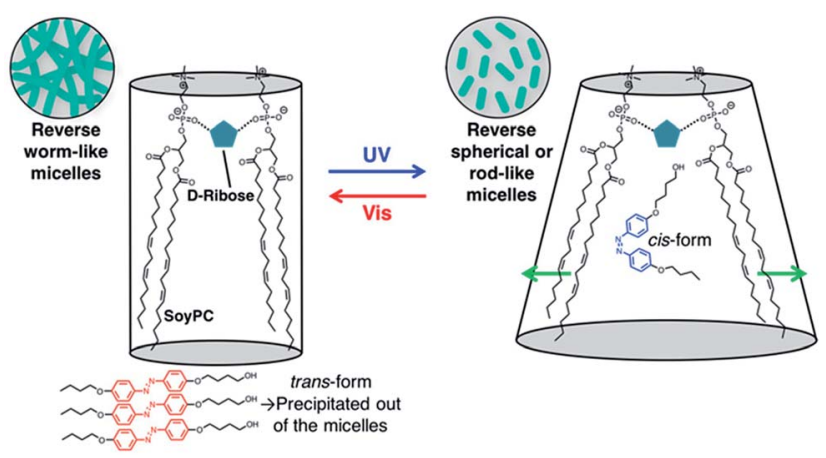

Fig. 5 A plausible mechanism of the morphological variations in structure of reverse micelles induced by UV and subsequent visible light irradiation. wormlike micelles is retained since $\mathbf{2}$ is less bulky and has weak interaction with the head group of SoyPC. The molecular length of trans-2 according to the stabilized structure ( $c a .22 \AA)$ matches one of SoyPC molecule. The low solubility of 2 in the decane solution also suppresses change in formation of the reverse wormlike micelles. Irradiation with UV light triggers the trans-to-cis isomerization and enhances the solubility of the azobenzene derivative into the micelles. When cis-2 bearing the bulky azobenzene moiety and mismatched molecular length (ca. $14 \AA$ ) with one of SoyPC is incorporated in the reverse wormlike micelles, the mean volume of the hydrophobic part of SoyPC is increased and the morphological transformation of the micelles occurs (Fig. 5, right). Infrared absorption spectra of the mixture with 2 shows that UV irradiation produces no shift in the peak position of the $\mathrm{P}=\mathrm{O}$ stretching vibration indicating that the hydrogen bond between the SoyPC headgroup and Dribose in the hydrophilic region of the micelles remains (Fig. S4 $\dagger$ ). These data support incorporation of the cis form of azobenzene in the hydrophobic rather hydrophilic region of the micelles. These results demonstrate that variations in solubility into the micelles formed by 2 produced by light irradiation, and appropriate hydrophobicity generates significant, reversible variations in the solution viscosity of lecithin-based reverse wormlike micelles.

\section{Conclusions}

We have demonstrated that photoirradiation induces significant and reversible variations in the solution viscosity of reverse wormlike micelles. Addition of photochromic azobenzene derivatives bearing butoxy and hydroxybutyl moieties to a Dribose/SoyPC/decane mixture forms reverse wormlike micelles. UV light irradiation induces a 150 -fold decrease in solution viscosity. This change is reversible upon subsequent irradiation with visible light. By screening azobenzene derivatives with polar or apolar substituents, the azobenzene analogue having appropriate hydrophobicity and variations of solubility into the micelles with photoirradiation produce the largest variations in solution viscosity. This wormlike micellar solution exhibiting photo-switchable solution viscosity can be applied to cosmetics, personal care products, and device components such as a heattransfer regulator. 


\section{Conflicts of interest}

There are no conflicts to declare.

\section{References}

1 J. Yang, Curr. Opin. Colloid Interface Sci., 2002, 7, 276-281.

2 G. Palazzo, Soft Matter, 2013, 9, 10668-10677.

3 P. Brown, C. P. Butts and J. Eastoe, J. Mater. Chem., 2013, 9, 2365-2374.

4 J. Eastoe, M. S. Dominguez, P. Wyatt, A. Beeby, D. H. U. Kingdom and R. K. Heenan, Langmuir, 2002, 18, 7837-7844.

5 F. P. Hubbard, G. Santonicola, E. W. Kaler and N. L. Abbott, Langmuir, 2005, 21, 6131-6136.

6 H. Sakai, A. Matsumura, S. Yokoyama, T. Saji and M. Abe, Langmuir, 1999, 103, 10737-10740.

7 Y. Orihara, A. Matsumura, Y. Saito, N. Ogawa, T. Saji, A. Yamaguchi, H. Sakai and M. Abe, Langmuir, 2001, 17, 6072-6076.

8 A. Matsumura, K. Tsuchiya, K. Torigoe, K. Sakai, H. Sakai and M. Abe, Langmuir, 2011, 27, 1610-1617.

9 M. Akamatsu, P. A. Fitzgerald, M. Shiina, T. Misono, K. Tsuchiya, K. Sakai, M. Abe, G. G. Warr and H. Sakai, J. Phys. Chem. B, 2015, 119, 5904-5910.

10 H. Sakai, Y. Orihara, H. Kodashima, A. Matsumura, T. Ohkubo, K. Tsuchiya and M. Abe, J. Am. Chem. Soc., 2005, 127, 13454-13455.

11 A. Matsumura, K. Sakai, H. Sakai and M. Abe, J. Oleo Sci., 2011, 60, 203-207.
12 H. Sakai, S. Taki, K. Tsuchiya, A. Matsumura, K. Sakai and M. Abe, Chem. Lett., 2012, 41, 247-248.

13 H. Shi, Y. Wang, B. Fang, X. Y. Talmon, W. Ge, S. R. Raghavan and J. L. Zakin, Langmuir, 2011, 27, 58065813.

14 H. Shi, W. Ge, H. Oh, S. M. Pattison, J. T. Huggins, Y. Talmon, D. J. Hart, S. R. Raghavan and J. L. Zakin, Langmuir, 2013, 29, 102-109.

15 H. Lee, K. K. Diehn, K. Sun, T. Chen and S. R. Raghavan, J. Am. Chem. Soc., 2011, 133, 8461-8463.

16 R. Kumar, A. M. Ketner and S. R. Raghavan, Langmuir, 2010, 26, 5405-5411.

17 R. G. Shrestha, N. Agari, K. Tsuchiya, K. Sakamoto, K. Sakai, M. Abe and H. Sakai, Colloid Polym. Sci., 2014, 292, 15991609.

18 D. Yang and J. Zhao, Soft Matter, 2016, 12, 4044-4051.

19 K. Hashizaki, H. Taguchi and Y. Saito, Chem. Lett., 2009, 38, 1036-1037.

20 E. Verploegen, J. Soulages, M. Kozberg, T. Zhang, G. Mckinley and P. Hammond, Angew. Chem., Int. Ed., 2009, 48, 3494-3498.

21 M. Ito, T. X. Wei, P.-L. Chen, H. Akiyama, M. Matsumoto, K. Tamada and Y. Yamamoto, J. Mater. Chem., 2005, 15, 478-483.

22 C. A. Dreiss, Soft Matter, 2007, 3, 956-970.

23 M. R. Han, D. Hashizume and M. Hara, New J. Chem., 2007, 31, 1746-1750.

24 X. Fu, Y. Yue, R. Guo, L. Li, W. Sun, C. Fang and C. Xu, CrystEngComm, 2009, 11, 2268-2271. 